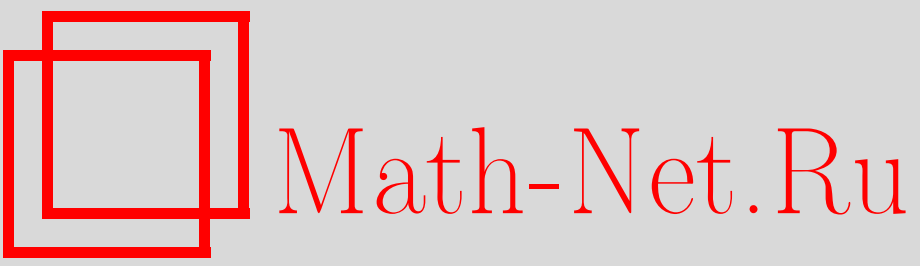

С. Н. Лакаев, С. М. Саматов, Условия конечности дискретного спектра гамильтониана системы трех произвольных частиц на решетке, УМH, 2002, том 57, выпуск 1, 155-156

DOI: https://doi.org/10.4213/rm482

Использование Общероссийского математического портала Math-Net.Ru подразумевает, что вы прочитали и согласны с пользовательским соглашением

http://www.mathnet.ru/rus/agreement

Параметры загрузки:

IP: 54.224 .187 .69

26 апреля 2023 г., 07:29:15 


\title{
УСЛОВИЯ КОНЕЧНОСТИ ДИСКРЕТНОГО СПЕКТРА ГАМИЛЬТОНИАНА СИСТЕМЫ ТРЕХ ПРОИЗВОЛЬНЫХ ЧАСТИЦ НА РЕШЕТКЕ
}

\author{
С. Н. ЛАКАев, С. М. Саматов
}

Конечность дискретного спектра для трехчастичного непрерывного оператора Шрёдингера изучены во многих работах [1]-[3]. Некоторые условия конечности числа связанных состояний вне существенного спектра гамильтониана системы трех одинаковых частиц установлены в [4].

В настоящей работе установлены условия конечности числа связанных состояний вне существенного спектра для гамилтониана системы трех произвольных частиц, взаимодействующих с помощью парных контактных потенциалов притяжения на решетке. Описана структура и местоположения существенного спектра.

Пусть $\mathbb{T}^{3}$ - трехмерньй тор, $L_{2}\left(\left(\mathbb{T}^{3}\right)^{3}\right)$ - гильбертово пространство квадратично интегрируемых функций, определенных на $\left(\mathbb{T}^{3}\right)^{3}$.

В импульсном представлении гамильтониан $H$ системы трех произвольных квантовых частиц действует в гильбертовом пространстве $L_{2}\left(\left(\mathbb{T}^{3}\right)^{3}\right)$ и имеет вид:

$$
\begin{aligned}
& (H f)\left(k_{1}, k_{2}, k_{3}\right)=\left(H_{0} f\right)\left(k_{1}, k_{2}, k_{3}\right) \\
& \quad-\sum_{\alpha=1 ; \alpha \neq \beta, \gamma}^{3} \mu_{\alpha} \iiint \delta\left(k_{\alpha}-k_{\alpha}^{\prime}\right) \delta\left(k_{\beta}+k_{\gamma}-k_{\beta}^{\prime}-k_{\gamma}^{\prime}\right) f\left(k_{1}^{\prime}, k_{2}^{\prime}, k_{3}^{\prime}\right) d k_{1}^{\prime} d k_{2}^{\prime} d k_{3}^{\prime} .
\end{aligned}
$$

Здесь $\left(H_{0} f\right)\left(k_{1}, k_{2}, k_{3}\right)=\left(\varepsilon_{1}\left(k_{1}\right)+\varepsilon_{2}\left(k_{2}\right)+\varepsilon_{3}\left(k_{3}\right)\right) f\left(k_{1}, k_{2}, k_{3}\right), \varepsilon_{\alpha}(p)=\ell_{\alpha} \sum_{i=1}^{3}\left(1-\cos p^{(i)}\right)$, $p=\left(p^{(1)}, p^{(2)}, p^{(3)}\right) \in \mathbb{T}^{3}, \mu_{\alpha} \in \mathbb{R}$ - энергия взаимодействия частиц $\beta$ и $\gamma, \delta(k)$ - трехмерная дельта-функция Дирака, $m_{\alpha}=\left(\ell_{\alpha}\right)^{-1}$ - масса частицы; индексы $\alpha, \beta$ и $\gamma$ различны и принимают значения $1,2,3$. Интеграл без указания пределов всюду означает интегрирование по всему тору $\mathbb{T}^{3}$

Пусть $K=k_{1}+k_{2}+k_{3}$-полный квазиимпульс системы трех частиц.

Если перейти к переменным $K=k_{1}+k_{2}+k_{3}, k_{1}=p, k_{2}=q$, то оператор $H$ является прямым операторным интегралом семейства операторов $\left\{H(K), K \in \mathbb{T}^{3}\right\}$, действующих при каждом $K \in \mathbb{T}^{3}$ в пространстве $L_{2}\left(\left(\mathbb{T}^{3}\right)^{2}\right)$ по формуле (см. [5])

$(H(K) f)(p, q)=\left(H_{0}(K) f\right)(p, q)-\mu_{1} \int f\left(p, q^{\prime}\right) d q^{\prime}-\mu_{2} \int f\left(q^{\prime}, q\right) d q^{\prime}-\mu_{3} \int f\left(q^{\prime}, p+q-q^{\prime}\right) d q^{\prime}$, где $\left(H_{0}(K) f\right)(p, q)=\varepsilon_{K}(p, q) f(p, q), \varepsilon_{K}(p, q)=\varepsilon_{1}(p)+\varepsilon_{2}(q)+\varepsilon_{3}(K-p-q), K \in \mathbb{T}^{3}$. Рассмотрим гамильтониан $H_{\alpha}(K)$, соответствующий системе трех частиц, где взаимодействуют только частицы $\beta$ и $\gamma$, действующий в гильбертовом пространстве $L_{2}\left(\left(\mathbb{T}^{3}\right)^{2}\right)$ по формуле

$$
H_{\alpha}(K) f(p, q)=\varepsilon_{\alpha \beta}(K ; p, q) f(p, q)-\mu_{\alpha} \int f\left(p, q^{\prime}\right) d q^{\prime},
$$

где $\varepsilon_{\alpha \beta}(K ; p, q)=\varepsilon_{\alpha}(p)+\varepsilon_{\beta}(q)+\varepsilon_{\gamma}(K-p-q), K \in \mathbb{T}^{3}$. Оператор $H_{\alpha}(K)$ разлагается в прямой операторньй интеграл семейства операторов $\left\{H_{\alpha}(K, p), p \in \mathbb{T}^{3}\right\}$, действующих при каждых $K, p \in \mathbb{T}^{3}$ в пространстве $L_{2}\left(\mathbb{T}^{3}\right)$ по формуле (см. [5]) $H_{\alpha}(K, p) f(q)=\varepsilon_{\alpha \beta}(K ; p, q) f(q)-$ $\mu_{\alpha} \int f\left(q^{\prime}\right) d q^{\prime}$.

Пусть $m_{K}=\min _{p, q \in \mathbb{T}^{3}} \varepsilon_{K}(p, q), M_{K}=\max _{p, q \in \mathbb{T}^{3}} \varepsilon_{K}(p, q)$. Обозначим

$$
\begin{gathered}
\varepsilon_{\min }^{(\alpha)}(K, p)=\min _{q \in \mathbb{T}^{3}} \varepsilon_{\alpha \beta}(K ; p, q), \quad \mu_{0}^{(\alpha)}(K)=\max _{p \in \mathbb{T}^{3}}\left(\int \frac{d q}{\varepsilon_{\alpha \beta}(K ; p, q)-\varepsilon_{\min }^{(\alpha)}(K, p)}\right)^{-1}, \\
\mu_{\min }^{(\alpha)}(K)=\max _{p \in \mathbb{T}^{3}}\left(\int \frac{d q}{\varepsilon_{\alpha \beta}(K ; p, q)-m_{K}}\right)^{-1} .
\end{gathered}
$$

Работа выполнена при поддержке ГКНТ Республики Узбекистан. 
ОПРЕДЕлЕниЕ. Если уравнение $H_{\alpha}(K, p) \psi(q)=\varepsilon_{\min }^{(\alpha)}(K, p) \psi(q), K, p \in \mathbb{T}^{3}$, имеет нетривиальное решение в пространстве $L_{\tau}\left(\mathbb{T}^{3}\right)$ при некотором $\tau \in[1,3 / 2)$, то говорят, что оператор $H_{\alpha}(K, p)$ имеет виртуальый уровень на левом крае непрерывного спектра (см. [3], [5]).

Пусть $G_{\mu_{\alpha}}$ - некоторая область и $\Gamma_{\mu_{\alpha}}-$ граница замкнутого множества $\mathbb{T}^{3} \backslash G_{\mu_{\alpha}}$.

ЛЕмма. а) Пусть $\mu_{\alpha} \in\left(0, \mu_{0}^{(\alpha)}(K)\right)$. Тогда для любого $K \in \mathbb{T}^{3}$ существует область $G_{\mu_{\alpha}} \subset \mathbb{T}^{3}$ и при всех $p \in G_{\mu_{\alpha}}$ оператор $H_{\alpha}(K, p)$ имеет единственное собственное значение $E_{\mu_{\alpha}}^{(\alpha)}(K, p)$, причем $0<E_{\mu_{\alpha}}^{(\alpha)}(K, p)<\varepsilon_{\min }^{(\alpha)}(K, p)$.

б) Пусть $\mu_{\alpha}=\mu_{0}^{(\alpha)}(K)$. Тогда для любого $K \in \mathbb{T}^{3}$ оператор $H_{\alpha}(K, 0)$ имеет виртуальный уровень на левом краю непрерывного спектра и для любого $p \in \mathbb{T}^{3} \backslash\{0\}$ оператор $H_{\alpha}(K, p)$ имеет единственное собственное значение $E_{\mu_{\alpha}}^{(\alpha)}(K, p)$ и $0<E_{\mu_{\alpha}}^{(\alpha)}(K, p)<$ $\varepsilon_{\min }^{(\alpha)}(K, p)$.

в) Пусть $\mu_{\alpha} \in\left(\mu_{0}^{(\alpha)}(K), \mu_{\min }^{(\alpha)}(K)\right]$. Тогда для любого $K \in \mathbb{T}^{3}$ существует область $G_{\mu_{\alpha}}$ и при всех $p \in G_{\mu_{\alpha}}$ оператор $H_{\alpha}(K, p)$ имеет единственное собственное значение $E_{\mu_{\alpha}}^{(\alpha)}(K, p)$, причем $E_{\mu_{\alpha}}^{(\alpha)}(K, p)<0$. При всех $p \in \mathbb{T}^{3} \backslash G_{\mu_{\alpha}}$ оператор $H_{\alpha}(K, p)$ имеет единственное собственное значение $E_{\mu_{\alpha}}^{(\alpha)}(K, p)$ и $0<E_{\mu_{\alpha}}^{(\alpha)}(K, p)<\varepsilon_{\min }^{(\alpha)}(K, p)$.

г) $E_{\mu_{\alpha}}^{(\alpha)}(K, p)=0$ есть виртуальный уровень оператора $H_{\alpha}(K, p)$ при всех $p \in \Gamma_{\mu_{\alpha}}$.

д) Пусть $\mu_{\alpha}>\mu_{\min }^{(\alpha)}(K)$. Тогда для любьх $K, p \in \mathbb{T}^{3}$ оператор $H_{\alpha}(K, p)$ имеет единственное отрицательное собственное значение $E_{\mu_{\alpha}}^{(\alpha)}(K, p)<0$.

Пусть $\operatorname{Im} E_{\mu_{\alpha}}^{(\alpha)}\left(K, G_{\mu_{\alpha}}\right)$ - образ множества $G_{\mu_{\alpha}}$ относительно отображения $E_{\mu_{\alpha}}(K)$, а $e_{\mu_{\alpha}}^{(\alpha)}(K)\left(E_{\mu_{\alpha}}^{(\alpha)}(K)\right)$ - его нижняя (соответственно верхняя) грань и $e(K)=\min _{\alpha} e_{\mu_{\alpha}}^{(\alpha)}(K)$.

Теорема 1. а) Пусть для любого $\alpha=1,2,3$ выполнены неравенства $0<\mu_{\alpha}<$ $\mu_{0}^{(\alpha)}(K)$. Тогда существенный спектр $\sigma_{\mathrm{ess}}(H(K))$ оператора $H(K)$ состоит из отрезка $\left[m_{K}, M_{K}\right]$.

б) Пусть для любого $\alpha=1,2,3$ имеют место неравенства $\mu_{0}^{(\alpha)}(K)<\mu_{\alpha}<\mu_{\min }^{(\alpha)}(K)$. Тогда $\sigma_{\mathrm{ess}}(H(K))$ состоит из отрезка $\left[e(K), M_{K}\right]$.

в) Пусть для любого $\alpha=1,2,3$ выполнены неравенства $\mu_{\alpha}>\mu_{\min }^{(\alpha)}(K)$. Тогда

$$
\sigma_{\mathrm{ess}}(H(K))=\bigcup_{\alpha=1}^{3} \sigma\left(H_{\alpha}(K)\right)=\bigcup_{\alpha=1}^{3}\left[e_{\mu_{\alpha}}^{(\alpha)}(K), E_{\mu_{\alpha}}^{(\alpha)}(K)\right] \cup\left[m_{K}, M_{K}\right]
$$

Обозначим через $W_{\delta}(0) \subset \mathbb{T}^{3}$ некоторую достаточно малую $\delta$-окрестность нуля.

Теорема 2. Если для некоторых $\alpha$ и $\beta$ выполнены неравенства $\mu_{\alpha} \neq \mu_{0}^{(\alpha)}(K)$ u $\mu_{\beta} \neq \mu_{0}^{(\beta)}(K)$ при $K \in W_{\delta}(0)$, то дискретный спектр оператора $H(K)$ разве лишь конечен.

ЗАмечАНИЕ. Отметим, что если $\mu_{\alpha}=\mu_{0}^{(\alpha)}(K), \mu_{\beta}=\mu_{0}^{(\beta)}(K), \mu_{\gamma} \in\left(0, \mu_{0}^{(\gamma)}(K)\right]$, то дискретный спектр оператора $H(K)$ бесконечен.

\section{СПИСОК ЛИТЕРАТУРЫ}

[1] Г. М. ЖКислин // Докл. АН СССР. 1972. Т. 207. №1. С. 25-28. [2] М. Рид, Б. Саймон. Методы современной математической физики. Т. 4. М.: Мир, 1982. [3] Д. К. Яфраев // ТМФ. 1975. Т. 25. С. 185-195. [4] ЖК. И. Абдуллаев, С. Н. Лакаев // ТМФ. 1997. Т. 111. № 1. С. 94. [5] С. Н. Лакаев // Функц. анализ и его прил. 1993. Т. 27. № 3. С. 15-28. 